\title{
Special issue on broadband mobile communications at very high speeds
}

\author{
Pingzhi Fan ${ }^{1 *}$, Erdal Panayirci ${ }^{2}, \mathrm{H}$ Vincent Poor ${ }^{3}$ and P Takis Mathiopoulos ${ }^{4,5}$
}

Future mobile communication systems aim at providing very high-speed data transmission, even under very high mobility scenarios such as high speed wheel-track trains (up to $574.8 \mathrm{~km} / \mathrm{h}$ test speed or $380 \mathrm{~km} / \mathrm{h}$ commercial speed), maglev trains (up to $581 \mathrm{~km} / \mathrm{h}$ test speed or $431 \mathrm{~km} / \mathrm{h}$ commercial speed), airplanes (about 400$1000 \mathrm{~km} / \mathrm{h}$ commercial speed), guided missiles (about $980-20,000 \mathrm{~km} / \mathrm{h}$ ) or spacecraft (at least $28,440 \mathrm{~km} / \mathrm{h}$ to remain on an earth orbit, at least $40,320 \mathrm{~km} / \mathrm{h}$ to leave earth). A related and particularly important commercial application is the strong worldwide increasing demand for broadband wireless communications in high speed railways to provide information and onboard entertainment services to passengers, train control, train dispatch, train sensor status transmission, video surveillance, etc.

Consequently, increasing demand on data rates to support broadband high speed communication systems in the presence of frequency selective fading channels with very high mobilities has resulted in research on designing computationally efficient yet faster new algorithms for channel estimation, equalization and detection, as well as fast handover, location update, modeling of rapidly time-varying channels, fast power control and dedicated network architectures, etc. Orthogonal frequency-division multiplexing (OFDM) is becoming a backbone structure of such systems, being standardized as the IEEE's 802.16 family - better known as Mobile Worldwide Interoperability Microwave Systems for Next-Generation Wireless Communication Systems (WiMAX) - and by the Third-Generation Partnership Project (3GPP) in the form of its Long-Term Evolution (LTE) project. Both systems employ orthogonal frequency division multiplexing/multiple access (OFDMA) as well as a new single-carrier frequency-division multiple access (SC-FDMA) format. To promote the IEEE 802.16 standards, recently, a high mobility feature has been introduced (IEEE $802.16 \mathrm{~m}$ ) to enable mobile broadband

\footnotetext{
* Correspondence: pzfan@swjtu.edu.cn

${ }^{1}$ Institute of Mobile Communications, Southwest Jiaotong University, Chengdu, Sichuan 610031, China

Full list of author information is available at the end of the article
}

services at vehicular speeds beyond $120 \mathrm{~km} / \mathrm{h}$. Since the signal transmission under very high speed scenarios will inevitably experience serious deterioration, it is imperative to develop key broadband mobile communication techniques for such very high speed vehicles.

This special issue aims at putting together the major achievements and developments in this field. There are 18 papers in this special issue, which have been organized into three thematic groups. The first group of five papers deal with the rapidly time-varying channel modeling and estimation, the next group of seven papers address data transmission under high mobility scenarios, and the last group of six papers are related to fast handover schemes, location prediction, etc.

Among the five papers dealing with channel modeling and estimation, two papers are concerned with channel estimation methods, namely, "Doubly Selective Channel Estimation for Orthogonal Frequency Division Multiplexing (OFDM) Modulated Amplify-and-Forward Relay Networks Using Superimposed Training" (Zhang Han), and "Channel Estimation in OFDM Systems Operating under High Mobility Using a Wiener Filter Combined with a Basis Expansion Model" (Ke Zhong, et al.). The former proposes a novel superimposed training (ST) strategy that allows the destination node to separately obtain the channel information of the source-relay link and the relay-destination link, from which the optimal ST signals are derived by minimizing the channel mean-square-error; and the latter proposes to combine the Wiener filter (WF) with a basis expansion model (BEM) to deal with channel estimation in various mobile environments, especially in high speed cases. The third paper, "A Highly Efficient Channel Sounding Method Based on Cellular Communications for HighSpeed Railway Scenarios" (Liu Liu, et al.), proposes an efficient channel sounding method based on a channel data recorder, by making use of the real time-dispersive channel measurements conducted along Zhengzhou-Xi'an (ZX) and Beijing-Tianjin (BT) high speed railways. The fourth paper, "Iterative Equalization for OFDM Systems over Wideband MSML Channels" (Tao Xu et al.), seeks to quantify the 
amount of interference resulting from wideband OFDM channels, which are assumed to follow the multi-scale multi-lag (MSML) model, then a conjugate gradient (CG) algorithm is used to equalize the channel iteratively. In the last paper of this group,"Frequency Tracking by the Method of Least Squares Combined with Channel Estimation for OFDM over Mobile Wireless Channels" (Rainfield Y Yen, et al.), rather than a common maximum-likelihood (ML) frequency tracking algorithm, least squares (LS) using repeated OFDM training blocks is employed which requires no channel knowledge, and can not only circumvent complications of ML estimation, but also obviates the need for the lengthy adaptive iteration process of joint estimation.

Concerning the seven papers related to the topic of data transmission under high mobility scenarios, the first paper, "Effect of Power and Rate Adaptation on the Spectral Efficiency of an MQAM/OFDM System under Very Fast Fading Channels" (Zhicheng Dong, et al.), proposes an adaptive MQAM/OFDM system under fast fading channels, resulting in substantial gains in spectral efficiency over the non-adaptive counterparts with perfect channel state information (CSI) or moderately imperfect CSI, and derives lower bounds on the maximum spectral efficiency and a closed-form expression for the average spectral efficiency of the proposed adaptive OFDM system. In the paper "Optimal Pilot Symbol Power Allocation under Time-variant Channels" (Michal Simko, et al.), the issue of how to distribute available power between data symbols and pilot symbols over time-varying channels is considered in order to maximize the overall throughput by choosing the post-equalization signalto-interference-plus-noise ratio (SINR) as the cost function. As a non-coherent transmission scheme that does not require CSI at either transmitters or receivers, unitary spacetime modulation is a promising technique that can be applied in high mobility scenarios where the fading channel parameters are changing too rapidly to be tracked and estimated, which is the emphasis of the paper "Multi-cell Cooperative Transmission Based on Unitary Space-time Modulation" (Heng Liu, et al.). The next paper, "Successive Interference Cancellation and MAP Decoding for Mobile MIMO OFDM Systems and Their Convergence Behavior" (Vamadevan Namboodiri, et al.). presents a suboptimal, successive interference cancellation (SIC) method based on maximum a posteriori probability (MAP) decoding over doubly dispersive channels for OFDM MIMO systems. With the appropriate use of the network topology, predictable location and speed information, Opportunistic beamforming (OBF) with dumb antennas, which does not require channel feedback, is useful for high speed railway applications. In the paper "Location Information-assisted Opportunistic Beamforming in LTE Systems for High-speed Railway" (Meng Cheng, et al.), two algorithms that can improve OBF system performance significantly are presented.
The sixth paper, "On the Applicability of Steerable Beams in LTE-Advanced Networks with High User Mobility" (Christos Papathanasiou, et al.), deals with the problem of applying scanning narrow beams to LTE-Advanced networks with fast moving mobiles, aiming at providing low complexity hardware implementation and low power consumption. The last paper in this group, "Realizing Broadband Wireless Communication for High Speed Trains" (Qinglin Luo, et al.), proposes a novel cloud-based high speed train communication (C-HSTC) system framework, featuring a new virtualized single cell design that mitigates the impact of conventional handover failure and guarantees continuous services. In conjunction with this design it also proposes a highly efficient joint transmit beamforming algorithm targeted at compensating the inter-carrier interference (ICI) caused by Doppler frequency shift due to mobility.

The six remaining papers deal with several fast handover schemes and other topics. The paper "A CoMP Soft Handover Scheme for LTE Systems in High Speed Railway" (Wantuan Luo, et al.), proposes an optimized handover scheme, in which the coordinated multiple point transmission technology and dual vehicle station coordination mechanism are applied to improve the traditional hard handover performance of LTE. In networks with highly mobile users, the inter-relay handoffs can occur very frequently, thus the paper "Inter-relay Handoff in Two-hop Relaying Networks with Highly Mobile Vehicles" (Di Wu, et al.), studies the inter-relay handoff decision problem in a two-hop cellular network with highly mobile vehicles using a semi-Markov decision process (SMDP) and taking into consideration speed and other factors. The next paper, "The Cross Layer RMPA Handover: A Reliable Mobility Pattern Aware Handover Strategy for Broadband Wireless Communications in the High Speed Railway Domain" (Marina Aguado, et al.), proposes an improved handover strategy for railway applications, the RMPA handover, an IEEE 802.16 handover strategy but specially "customized" and enhanced for high speed mobility scenarios. As transport layer (L4 in ISO layers) protocols such as stream control transmission protocols can support seamless handover in high-speed mobility scenarios, the paper "Adaptive Transport Layer Protocol for Highly Dynamic Environments" (Hala Eldaw Idris Jubara, et al.), discusses an adaptive transport layer protocol in mobile WiMAX networks supporting seamless handover and guaranteeing quality-of-service (QoS) for rapid handover rates. The last two papers deal with location prediction and connectivity analysis in high speed vehicular ad-hoc networks (VANETs). In particular, the paper "A Novel Vehicular Location Prediction Based on Mobility Patterns for Routing in Urban VANETs" (Guangtao Xue, et al.), proposes a novel approach by adopting a variable-order Markov model to abstract vehicular 
mobility pattern (VMP) from real trace data in Shanghai for predicting the possible trajectories of moving vehicles. To handle the rapidly moving inter-vehicle information exchange within limited radio ranges and self-organize VANETs, the last paper, "Available Connectivity Analysis under Free Flow State in VANETs" (Chen Chen, et al.), is concerned with the connectivity issue, both one-hop direct connectivity and multi-hop indirect connectivity by defining a new metric termed "available connectivity".

\section{Competing interests}

The authors declare that they have no competing interests.

\section{Authors' information}

Guest Editors: Pingzhi Fan, Erdal Panayirci, H. Vincent Poor, P. Takis Mathiopoulos

Prof. Pingzhi Fan received his M.S. degree in Computer Engineering from the Southwest Jiaotong University, PRC, in 1987, and Ph.D. degree in Electronic Engineering from the Hull University, U.K., in 1994. He is currently a professor and director of the institute of mobile communications,

Southwest Jiaotong University, China, and a guest professor of Leeds University, UK (1997-), a guest professor Shanghai Jiaotong University (1999-). He was a recipient of the UK ORS Award (1992), and the Outstanding Young Scientist Award (NSFC, 1998). He served as a general chair or TPC chair of a number of international conferences, and guest editor or editorial member of 5 international journals. He is the founding chair of IEEE VTS BJ Chapter and IEEE Chengdu Section. He also served as a board member of IEEE Region 10 and IET Asia-Pacific Region. He is the inventor of 20 patents, the author of over 380 research papers, and 8 books or edited books published by John Wiley \& Sons Ltd, RSP, IEEE Press, Springer, Nova Science, etc. His research interests include high mobility wireless communications, spreadspectrum and CDMA techniques, information theory \& coding, etc. Prof. Fan is a fellow of IET, CIE and CIC.

Prof. Erdal Panayirci received his Ph.D. degree in Electrical Engineering and System Science from Michigan State University, USA. He is currently Professor of Electrical Engineering and Head of the Electronics Engineering Department at Kadir Has University, Istanbul, Turkey. He published extensively in leading scientific journals and international conferences. He has co-authored the book Principles of Integrated Maritime Surveillance Systems (Boston, Kluwer Academic Publishers, 2000). Dr. Panayırcı spent the academic year 2008-2009, in the Department of Electrical Engineering, Princeton University, New Jersey, USA. He has been the principal coordinator of a 6th and 7th Frame European project called NEWCOM (Network of Excellent on Wireless Communications) and WIMAGIC Strep project representing Kadir Has University. Dr. Panayırcı was an Editor for IEEE Trans on Communications in the areas of Synchronization and Equalizations in 1995-1999. He served as a Member of IEEE Fellow Committee in 2005-2008. He was the Technical Program Chair of ICC'2006 and PIMRC'2010. Presently he is head of the Turkish Scientific Commission on Signals and Systems of URSI (International Union of Radio Science). Dr. Panayırci's recent research interests include communication theory, synchronization, advanced signal processing techniques and their applications to wireless communications, etc. He is an IEEE life fellow.

Prof. H. Vincent Poor received his Ph.D. in EECS in 1977 from Princeton University, where he is currently the Michael Henry Strater University Professor of Electrical Engineering, and Dean of the School of Engineering and Applied Science. He has also held visiting appointments at a number of universities and research institutions in the U.S. and abroad, including Imperial College (London), Stanford and Harvard. Dr. Poor is a member of the U.S. National Academy of Engineering and the U.S. National Academy of Sciences, a Fellow of the American Academy of Arts \& Sciences, and an International Fellow of the Royal Academy of Engineering of the U.K. He is also a Fellow of the IEEE, the Institute of Mathematical Statistics, the Optical Society of America, and other scientific and technical organizations. He has served as President of the IEEE Information Theory Society, and as Editor-inChief of the IEEE Transactions on Information Theory. He received a Guggenheim Fellowship in 2002 and the IEEE Education Medal in 2005. Recent recognition of his work includes the 2010 IET Ambrose Fleming Medal for Achievement in Communications, the 2011 IEEE Eric E. Sumner
Award, the 2011 Society Award of the IEEE Signal Processing Society, and honorary doctorates from Aalborg University, the Hong Kong University of Science and Technology, and the University of Edinburgh. His interests lie in the areas of stochastic analysis, statistical signal processing and information theory, and their applications in wireless networks and related fields. Prof. P. Takis Mathiopoulos received the Ph.D. degree in Electrical Engineering from the University of Ottawa, Canada, in 1989. He is currently Director of Research at the Institute for Space Applications and Remote Sensing (ISARS) of the National Observatory of Athens (NOA), where he has established the Wireless Communications Research Group. He was a faculty member (1989-2003) at the Department of Electrical and Computer Engineering (ECE) of the University of British Columbia (UBC), where he was promoted to Professor in 2000. He is also an elected Professor of Digital Communications at the Department of Informatics and Telecommunications, University of Athens. He has been the PI for more than 40 R\&D projects supported by Canadian, European and Greek funding agencies. He has published more than 200 papers in journals and international conference proceedings and is or has been on the Editorial board of many scientific journals, including the IEEE Transactions on Communications (1993 - 2005). His research interests are mainly dealing with the physical layer of digital communications for terrestrial and satellite based systems. Secondary research interests include remote sensing and GIS systems. He is an ASI Fellow, a Killam Research Fellow and a co-recipient of 2 best conference paper awards.

\section{Acknowledgments}

The guest editors would like to thank the authors of all submitted papers for considering our special issue to disseminate their work, and to all the reviewers for their conscientious work. Our special thank also goes to Bernardino McCartney of JWCN for excellent assistance in making this special issue possible. This work was partially supported by the National Basic Research Program (973 Program, No.2012CB316100), the National Science Foundation of China (NSFC No.61032002) and the Innovative Intelligence Base Project (111 project No.111-2-14).

\section{Author details}

'Institute of Mobile Communications, Southwest Jiaotong University, Chengdu, Sichuan 610031, China. ${ }^{2}$ Dept of Electrical and Electronic Engineering, Kadir Has University, Cibali Merkez Kampusu, Cibali, Istanbul 34083, Turkey. ${ }^{3}$ School of Engineering and Applied Science, Princeton University, C-230 Engineering Quadrangle, Olden Street Princeton, NJ 08544, USA. ${ }^{4}$ Institute for Space Applications and Remote Sensing, National Observatory of Athens, I. Metaxa \& Vas. Pavlou, Palaia Penteli, Athens, GR 15236, Greece. ${ }^{5}$ Department of Informatics and Telecommunications, University of Athens, Athens 15784, Greece.

Received: 19 August 2012 Accepted: 19 August 2012

Published: 31 August 2012

doi:10.1186/1687-1499-2012-279

Cite this article as: Fan et al:: Special issue on broadband mobile communications at very high speeds. EURASIP Journal on Wireless Communications and Networking 2012 2012:279.

\section{Submit your manuscript to a SpringerOpen ${ }^{\mathcal{D}}$ journal and benefit from:}

- Convenient online submission

Rigorous peer review

- Immediate publication on acceptance

- Open access: articles freely available online

- High visibility within the field

- Retaining the copyright to your article

Submit your next manuscript at $\gg$ springeropen.com 